\title{
Culture and Technologies for Social Interaction
}

\author{
Qinying Liao ${ }^{1}$, Susan R. Fussell ${ }^{2}$, Sheetal K. Agarwal ${ }^{3}$, Arun Kumar ${ }^{3}$, \\ Amit A. Nanavati ${ }^{3}$, Nitendra Rajput ${ }^{3}$, and Yingxin Pan $^{1}$ \\ ${ }^{1}$ IBM China Research Lab. Bldg. 19, Zhongguancun Software Park, \\ 100094 Beijing, P.R. China \\ \{liaoqy, panyingx $@_{\mathrm{Cn}}$. ibm. com \\ ${ }^{2}$ Cornell University, 336 Kennedy Hall, Ithaca, PA 14853 USA \\ suefussell@gmail.com \\ ${ }^{3}$ IBM India Research Lab., 4, Block - C, Institutional Area, \\ Vasant Kunj, New Delhi 110070 India \\ \{sheetaga, kkarun, namit, rnitendra\}@in.ibm.com
}

\begin{abstract}
While social activities and user requirements clearly vary across cultures, we are far from having a systematic understanding of culture's role in the design, development and use of social technologies. This workshop will bring together a mix of HCI researchers and practitioners, social scientists and psychologists who are interested in areas of social technologies and culture, in order to (a) examine the design and use of technologies for social interaction in different cultures to date; (b) explore various viewpoints on the key issues for future research; (c) outline various approaches and identify some basic guidelines for understanding cultural impacts in building social technologies as well as user interfaces and (d) foster new collaborations in the community.
\end{abstract}

Keywords: Culture, social interaction, social networking sites, online community, social technology, social software.

Recent years have witnessed an increase of social collaborative technologies worldwide, such as discussion groups, blogs, wikis, and social networking sites, resulting in the rapid development of online communities, where people communicate, share information and keep in touch with each other. Designing user interfaces for these technologies requires intensive attention on understanding social interactions among users and acknowledging demographic diversity. In today's global marketplace, these differences may reflect world-wide cultures. Despite its importance, however, most behavioral research on computer-mediated social interactions and many of the technologies developed to support these activities have to date been rooted in Western cultures.

This workshop attempts to bring together researchers, designers and developers with interests in understanding interactions between culture and social technologies. Through collaborative tasks and group discussions, we aim to (a) examine the design and use of technologies for social interaction in different cultures; (b) explore various viewpoints on the key issues for future research; (c) outline various approaches and identify some basic guidelines for understanding cultural impacts in building social technologies and user interfaces; and (d) foster new collaborations in the community. 
Previous research has indicated that culture does indeed influence acceptance of technology [2]. As for technologies that support social activities, such as social networking sites, understanding the impact of culture involves understanding the social behaviors themselves. Think about a social recommendation site where you find information about restaurants. Do you want to see the rankings first or read about the comments or look for the background information of the restaurant to assess its credibility? Different cultures may look for different data to make decisions.

Social research suggests that societies vary along a number of important dimensions that might be expected to influence how and why people use social technologies and what features might be most desirable and/or useful for them. For example, Kavan and Fussell et. al. [3] found that multi-party chat, audio-video chat and emoticons were much more popular in Asia than in North America, reflecting the differences between collectivistic, high-context cultures and individualistic, low-context cultures. Ardichvili et.al. [1] found that modesty requirements as well as high degree of competitiveness among employees were serious barriers to information sharing in China through online communities of practice, but not in Brazil and Russia.

These efforts, however, have to date been scattered and we are far from having a systematic understanding of the concept and role of culture in the use and design of online communities and supporting technologies. To cope with these challenges, this workshop will address multiple issues: (1) the first will be identification of cultureindependent and culture-dependent social interactions and technologies, to bring some order to the diverse studies on cultural aspect of social technologies and explore key issues for future research; (2) secondly, we will collectively examine theoretical and methodological challenges, such as the cultural factors to be considered during the design of social software, models required to identify and evaluate the culture's role in dynamic social interactions, measurement instruments and research design that can be developed to permit direct, cross-cultural comparisons, methods that cross-culture theories can be translated into usable features for social software, etc.; (3) we will also consider various approaches by which design recommendations for social interaction can be specified via the dialogues among technologies (developers), user interfaces (designers) and usage patterns (researchers).

Productive collaboration will be emphasized in the workshop through collaborative tasks and group discussions. By the end of the workshop, we will produce a poster for the INTERACT conference as well as a report for publication. A final outcome goal is to strengthen relationships among researchers in the area of culture and humancomputer interaction that may lead to new research collaborations. The major target will be the INTERACT, CHI, CSCW communities.

\section{References}

1. Ardichvili, A., Maurer, M., et al.: Cultural influences on knowledge sharing through online communities of practice. Journal of Knowledge Management, 10 (2006)

2. Evers, V., Day, D.: The role of culture in interface acceptance. In: Howard, S., Hammond, J., Lindgaard, G. (eds.) Human Computer Interaction: INTERACT 1997, Sydney. Chapman and Hall, Boca Raton (1997)

3. Kayan, S., Fussell, S.R., et al.: Cultural Differences in the Use of Instant Messaging in Asia and North America. In: CSCW 2006, Extended Abstracts (2006) 